\section{Severe scratcher-reaction: an unknown health hazard?}

\author{
Carsten Sauer Mikkelsen, ${ }^{1}$ \\ Helene Ringe Holmgren, ${ }^{2}$ \\ Kristian Bakke Arvesen, ${ }^{3}$ \\ Reem Dina Jarjis, ${ }^{4}$ \\ Gudjon Leifur Gunnarsson 5
}

'Private Practice, Broenderslev, Denmark;

2Private Practice, Frederikshavn,

Denmark; ${ }^{3}$ Department of

Dermatovenereology, Aarhus University

Hospital, Denmark; ${ }^{4}$ Department of

Plastic Surgery, Aalborg University

Hospital, Denmark; ${ }^{5}$ Department of

Plastic Surgery, Telemark Hospital, Skien, Norway

\section{Abstract}

Tattoos are well known to cause skin problems and the number of reported adverse reactions after tattooing has increased. Illegally imported tattoo ink is unrestrained and can contain unknown ingredients and contamination thereby posing a serious health hazard. We present a case illustrating the risk of pronounced phototoxic allergic reaction and other severe complications after using home kit tattoo ink.

\section{Introduction}

A 48-year-old Caucasian male developed an impetigonous rash followed by exfoliative dermatitis occurring after the use of a home kit tattoo ink imported from China via the internet. We suggest that a sudden severe phototoxic tattoo ink reaction ignited a cascade of interesting events that we feel obliged to report since we were unable to find any similar cases in the literature.

\section{Case Report}

This case concerned a man in his late 40's, otherwise healthy with no previously known illnesses or skin diseases. His medical history revealed earlier urticarial reaction to penicillin. A few weeks after being sun burned on his arms, the patient developed multiple bullae and itchiness on his left forearm. A few days later he developed an exuding erythema rash with crustations. The rash spread the next day to the other arm developing edema, redness and heat. Eventually the same rash spread to the neck and abdomen.

The patient contacted his general practitioner 14 days after the onset of symptoms and was then referred to the local hospital under suspicion of severe impetigo.

Upon his admission he was relatively unaffected, sub-febrile $\left(38^{\circ} \mathrm{C}\right)$ with extreme edema of his arms, generalized erythema and multiple fissures with pus formation and some areas with dry crustations (Figure 1).

Lab results showed slight leukocytosis 12 (3.5-10.0) with neutrofiles 9 (2.0-7.0) and marginally raised crp $22(<8 \mathrm{mg} / \mathrm{L})$.The initial diagnosis was impetigo and erysipelas and he was treated with intravenous Cefuroxim 750 $\mathrm{mg} \times 4$ daily. The itchiness was treated with fexofenadin $180 \mathrm{mg} \times 1$. The patient responded to the treatment and his condition improved rapidly. The cultivated swab taken from the forearm was positive for Staphylococcus aureus.

Due to the severity of skin symptoms a dermatologist was consulted and it became apparent that the patient had 2 months earlier had a tattoo on his left forearm. For this purpose the patient had ordered tattoo needles and ink over the internet from China. It was in this precise tattooed area that the symptoms with bullae development and impetigo arose.

The consultant dermatologist diagnosed the patient with staphylococcal scalded skin syndrome (SSSS), and began a 7 day treatment with prednisolone $37.5 \mathrm{mg} \times 1$ daily and a group III topical cortisone cream. The skin became smooth after a few days.

The treatment was founded on the probable phototoxic reaction to the tattoo ink followed by a break in the skin barrier due to itching and resulting in Staphylococcus aureus infection and later SSSS. There was also a speculation of the probability of contaminated tattoo ink but further investigations of other ink bottles from the same batch tested negative to microbes when cultured.

A few days after the completed prednisolone treatment, the patient developed universal exfoliative dermatitis, with exaggerated hyperkeratosis changes to the palms of the hands and soles of the feet (Figure 1). The patient was retreated with high dosage prednisolone, a group IV hormone cream and in addition ciclosporin $100 \mathrm{mg} \times 2$ daily. The response to this treatment was striking.

The patient's symptoms were most likely due to allergic contact dermatitis reaction with some contents of the tattoo ink. The biopsises concluded with photo-allergic dermatitis in early and late phase. Further investigations with light tests (UVA and UVB) and photopatch test were all negative.

The patient is still followed by the dermatology department, 6 months after the onset of symptoms and continues a ciclosporin treatment. At the latest control there were only
Correspondence: Carsten Sauer Mikkelsen, Private Practice, Bredgade 13, 9700 Broenderslev, Denmark.

Tel.: +45.201.00198.

E-mail: c.s.mikkelsen@hotmail.com

Key words: tattoo, ink, home kit, exfoliative dermatitis, impetigo.

Contributions: the authors contributed equally.

Conflict of interest: the authors declare no potential conflict of interest.

Received for publication: 17 January 2015.

Revision received: 19 March 2015.

Accepted for publication: 19 March 2015.

This work is licensed under a Creative Commons Attribution NonCommercial 3.0 License (CC BYNC 3.0).

(C) Copyright C.S. Mikkelsen et al., 2015

Licensee PAGEPress, Italy

Dermatology Reports 2015; 7:5816

doi:10.4081/dr.2015.5816

minor dermatitis changes found in the palms of the hands. The patient therefore has a good prognosis. A patch test with the ink contents would be ideal to study and maybe find which ingredients the patient reacted to but unfortunately we haven't been able to acquire such a sample.

\section{Discussion}

The number of tattooed people has substantially increased in the past years. Surveys in different countries reveal this to be up to $24 \%$ of the population. ${ }^{1}$ No reports are found about the number of people using home kit tattoo ink. Tattoos are well known to cause skin problems and the number of reported adverse reactions after tattooing has increased. This includes transient acute inflammatory reaction due to trauma of the skin with needles and medical complications such as superficial and deep local infections, systemic infections, allergic contact dermatitis, photodermatitis, granulomatous and lichenoid reactions and skin diseases localized on tattooed area (eczema, psoriasis, lichen rubor, and morphea). ${ }^{2}$ Tattoo colors consist of inorganic pigments, organic dyes, or a combination of both. In the past, it appears that heavy metals, that were the backbone of tattooing for decades, have been largely replaced by organic colorants. ${ }^{3}$ The reactions to tattoos are reported more often where the colors red and yellow give a serious allergic reaction and acute tattoo reactions are most commonly associated 
with red pigments. ${ }^{4,5}$ Reports on severe reactions to green pigments (especially due to chromium) is also described in the literature. ${ }^{6}$ The etiology is still uncertain but it is generally considered to be a delayed-type hypersensitivity reaction to either the pigment itself or its carrier solution. ${ }^{7}$ In our case both the light and photo-patch test wee negative. This is also the findings in a newer published study. ${ }^{8}$ After patch-testing patients with tattoo reactions, the study indicates that the putative allergen(s) causing tattoo reactions are formed inside the dermis and external factors like sunlightproduce photochemical cleavage of tattoo pigments in vivo in the skin and may contribute to allergen formation. This means that regular patch-testing will not reproduce the reaction due to inadequate penetration of the skin, and will therefore be negative.

Photoinduced reactions, principally associated with yellow pigments, are generally present as erythematous, pruriginous nodules that appear following sun exposure..$^{9}$ Very few reports describe phototoxic reaction to the green pigment as described in our case-story.

Recent analyses of a wide range of commercially available tattoo inks demonstrated surprisingly high rates of bacterial contamination in both open and unopened ink bottles. ${ }^{10,11}$ It wasn't possible to test the green ink bottle used by the patient in this case. But the rest of the imported inkbottles were tested for bacterial contamination without any positive findings. Our patient represents a pronounced phototoxic allergic reaction to the green ink in the home kit tattoos activated by sunburn. In this case we suggest that the Staphylococcus aureus infection and subsequent SSSS were self-induced based on scratching of the affected skin-area where the green tattoo was present on his forearm. Following remission of the bacterial infection our patient was strained by the chronic systematic allergic reaction which indicated the need for a long term oral immune suppression for over 6 months.

The effect of tattoo colors on the skin immune system is unknown. The tattoo colors are known to have a possible contamination of nanoparticles that are known to invade the lymphatic system and further into the center of the cells where they might affect cellular function. ${ }^{12}$ A large concentration of tattoo colors has been discovered in the lymph-node biopsies of tattooed patients in a project led by professor Jørgen Serup at the dermatology department in Bispebjerg hospital in Denmark. No one knows the long term effects of the accumulation of tattoo colors in the lymphatic system. Could it in any way hinder its normal function or by chronic inflammation increase the risk of later cancer developer other late complications? The potentially harmful ingredients in such substances, especially uncontrolled and illegally imported tattoo inks are

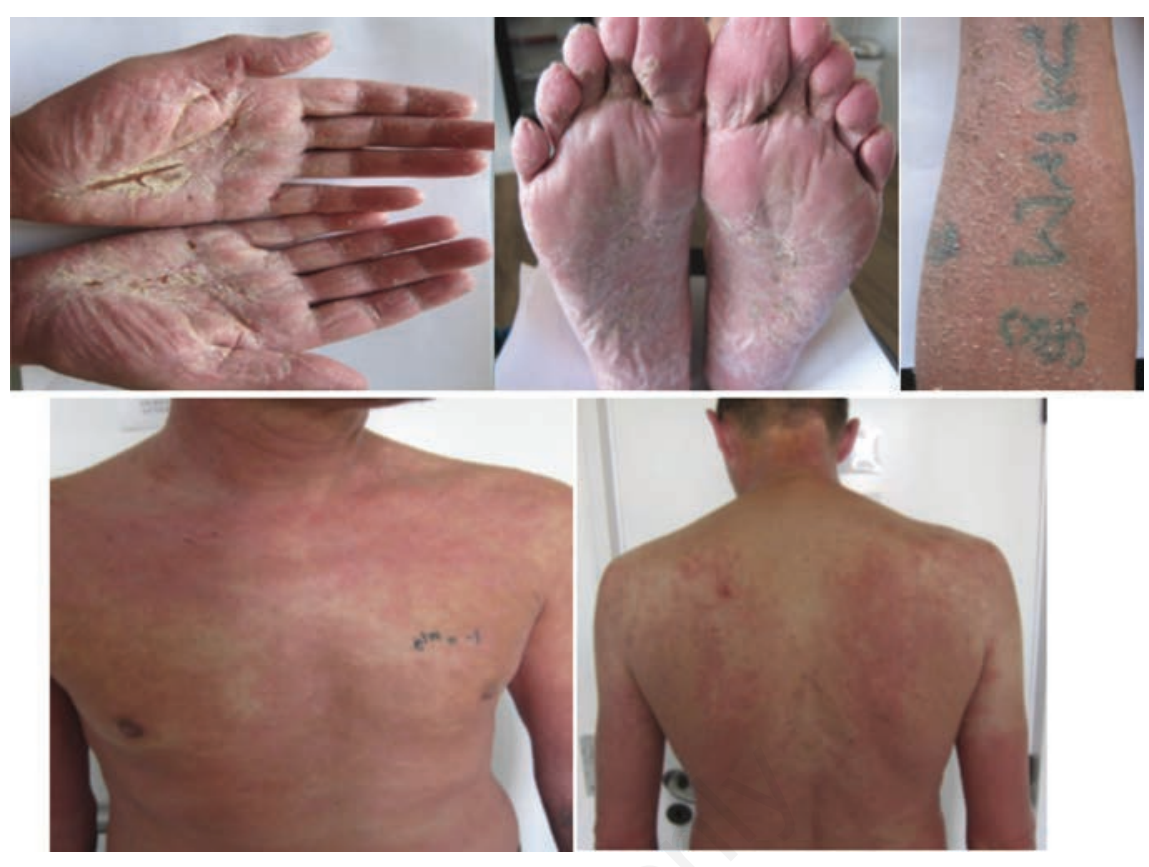

Figure 1. Images showing patient's edema of arms, generalized erythema and multiple fissures with pus formation and some areas with dry crustations.

completely unknown.

Stock bottles of tattoo ink may contain environmental bacteria and bacteria pathogenic to humans and packaging, labeling and preservation of inks have been shown to be inadequate. ${ }^{11-14}$ We fear the lack of safety and risk of cutaneous inoculation of dangerous bacteria and toxic allergens. Even the possibility of resistant nontuberculous mycobacteria or methicillin resistant Staphylococcus aureus strains and long term consequences of such practice. We have searched the literature and were unable to find other cases of SSSS and chronic systemic allergic reactions arising from home kit tattoos.

\section{Conclusions}

Illegally imported tattoo ink is uncontrolled and can contain practically anything and thereby pose serious health hazard as described in this case. Awareness needs to be raised of the risks involved.

\section{References}

1. Wenzel SM, Rittmann I, Landthaler M, et al. Adverse reactions after tattooing: review of the literature and comparison to results of a survey. Dermatology 2013:226:138-47.

2. Bassi A, Campolmi P, Cannarozzo G, et al.
Tattoo-associated skin reaction: the importance of an early diagnosis and proper treatment. Biomed Res Int 2014;2014:354608.

3. Sanghavi SA, Dongre AM, Khopkar US. Tattoo reactions - an epidemic on the surge: a report of 3 cases. Indian $\mathrm{J}$ Dermatol Venereol Leprol 2013;79:231-4.

4. Champman G, Thoroton-Hildyard CA. Two decades later: a delayed red ink tattoo reaction. BMJ Case Rep 2014;2014:bcr2013201726.

5. Cui Y, Spann AP, Couch LH, et al. Photodecomposition of pigment yellow 74, a pigment used in tattoo inks. Photochem Photobiol 2004:80:175-84.

6. Kaur RR, Kirby W, Maibach H. Cutaneous allergic reactions to tattoo inj. J Cosmet Dermatol 2009;8:295-300.

7. Sweeney SA, Hicks LD, Ranallo N, et al. Perforating granulomatous dermatitis reaction to exogenous tattoo pigment: a case report and review of the literature. Am J Dermatopathol 2013;35:754-6.

8. Serup J, Hutton Carlsen K. Patch test study of 90 patients with tattoo reactions: negative outcome of allergy patch test to baseline batteries and culprit inks suggests allergen(s) in the skin trough haptenization. Contact Dermatitis 2014;70:255-63.

9. Adams DR, Eid MP, Badreschia S, et al. Self assessment examination of the American Academy of Dermatology. A violaceus plaque. J Am Acad Dermatol 2006;54:185-7.

10. Baumgartner A, Gautsch S. Hygienicmicrobiological quality of tattoo and per- 
manent make-up colours. J Verbrauch Lebensm 2011:6:319-25.

11. Høgsbjerg T, Saunte DM, Serup J, et al. Microbial status and product labeling of 58 original tattoo inks. J Eur Acad Dermatol Venereol 2013;27:73-80.

12. Høgsberg T, Loeschner K, Serup J, et al.
Tattoo inks in general usage contain nanoparticles. $\mathrm{Br} \quad \mathrm{J}$ Dermatol 2011;165:1210-8.

13. Falsey RR, Kinzer $M H$, Hurst $S$, et al. Cutaneous inoculation of nontuberculous mycobacteria during professional tattooing: a case series and epidemiologic study.
Clin Infect Dis 2013;57:e143-7.

14. Centers for Disease Control and Prevention. Tattoo-associated nontuberculous skin infections: multiple states, 20112012. MMWR Morb Mortal Wkly Rep 2012;61:653-6. 